BULL. AUSTRAL. MATH. SOC.

VOL. 25 (1982), 44I-45I.

\title{
ISOMORPHISMS OF SOME SEGAL ALGEBRAS AND THEIR MULTIPLIER ALGEBRAS
}

\section{U.B, TEWARI AND K. Parthasarathy}

Let $G_{1}, G_{2}$ be locally compact groups and let $S_{1}, S_{2}$ be Segal algebras on $G_{1}, G_{2}$ respectively. Under certain conditions on $G_{1}, G_{2}$ and $S_{1}, S_{2}$, we prove that if there is a bipositive or isometric isomorphism between $S_{1}, S_{2}$ or between their multiplier algebras then $G_{1}$ and $G_{2}$ are topologically isomorphic.

\section{Introduction}

Let $G$ be a locally compact group with a fixed left haar measure $d x$. The topology of any locally compact group will be assumed to be Hausdorff. For a function $f$ on $G$, the left translate $x^{f}$ and the right translate $f_{x}$ of $f$ are defined by

$$
f(y)=f\left(x^{-1} y\right)
$$

and

$$
f_{x}(y)=f\left(y x^{-1}\right) \Delta\left(x^{-1}\right), x, y \in G
$$

where $\Delta$ is the modular function of $G$.

A dense subalgebra $S(G)$ of $L_{1}(G)$ is said to be a Segal algebra, if it satisfies the following conditions:

Received 6 January 1982. 
(1) $S(G)$ is a Banach space under a norm $\|\cdot\|_{S}$ such that $\|f\|_{S} \geq\|f\|_{1}$ for all $f \in S(G)$;

(2) $S(G)$ is left invariant $\left(f \in S(G) \Rightarrow y^{f} \in S(G)\right.$ for all $y \in G$ ) and for each $f \in S(G)$ the mapping $y \rightarrow y^{f}$ of $G$ into $S(G)$ is continuous;

(3) $\|f\|_{S}=\|f\|_{S}$ for all $f \in S(G)$ and $y \in G$.

If $G$ is discrete then every segal algebra $S(G)$ coincides with $L_{\perp}(G)$.

A Segal algebra $S(G)$ is said to be symmetric if $S(G)$ satisfies the following conditions:

(1') $S(G)$ is right invariant and for each $f \in S(G)$ the mapping $y+f_{y}$ of $G$ into $S(G)$ is continuous;

(2') $\left\|f_{y}\right\|_{S}=\|f\|_{S}$ for all $f \in S(G)$ and $y \in G$.

If $G$ is abelian then every Segal algebra $S(G)$ is symmetric.

A Segal algebra $S(G)$ is said to be pseudosymnetric if it satisfies $\left(I^{\prime}\right)$ and $S(G)$ contains functions $u \geq 0$ with $\int u d x=1$ and arbitrarily small support.

For various properties of Segal algebras, we refer to Reiter [13] and [12].

A multiplier $T$ of a Segal algebra $S$ on $G$ is a linear map from $S$ to $S$ such that $T(f * g)=T(f) * g$ for $f, g \in S$. It is well known that a multiplier $T$ of $S$ is a continuous linear operator on $S$. The set $M(S)$ of all multipliers of $S$ is a Banach algebra with multiplication as composition and the norm as operator norm. $M(S)$ is called the multiplier algebra of $S$.

The basic references for multipliers are Larsen [8] and Sections 35 and 36 of Hewitt and Ross [5].

Let $S$ be a Segal algebra on $G$ and let $M(G)$ denote the algebra of bounded regular Borel measures on $G . M(G)$ can be canonically imbedded 
in $M(S)$ by considering $\mu \in M(G)$ as a multiplier defined by $\mu(f)=\mu * f$, for all $f \in S$. This correspondence is norm decreasing; that is, $\|\mu\|_{M(S)} \leq\|\mu\|_{M(G)}$. For $S=L_{1}(G)$, this imbedding is an isometric isomorphism of $M(G)$ onto $M(S)$.

For $a \in G$, let $\delta_{a} \in M(G)$ denote the unit point mass at ' $a$ '. As an e:-ement of $M(S), \delta_{a}$ is nothing but left translation by ' $a$ ' ; that is, $\delta_{a}(f)=a_{a}^{f}$. By the definition of Segal algebras $\left\|_{\alpha} f\right\|_{S}=\|f\|_{S}$, for every $f \in S$. Hence $\lambda \delta_{a}$ for $|\lambda|=1$ and $a \in G$ is an isometric multiplier of $S$. The authors have proved in [1]] that for a large class of Segal algebras, these are the only isometric multipliers. This result assumes special significance in relation to Theorem 2 of this paper.

A multiplier $T$ of $S$ is said to be positive if $T f \geq 0$ almost everywhere whenever $f \geq 0$ almost everywhere and $f \in S$. If $\mu \in M(G)$ is a positive measure on $G$ then $\mu$ is a positive multiplier of $S$. Conversely, we shall prove in Lemma 1 that for a large class of Segal algebras these are the only positive multipliers. This result will be the key to the proof of Theorem 1 of this paper.

Let $G_{1}, G_{2}$ be locally compact groups and let $A_{1}, A_{2}$ be spaces of measurable functions on $G_{1}, G_{2}$ respectively. A mapping $\Psi: A_{1} \rightarrow A_{2}$ is said to be bipositive whenever $\Psi_{f} \geq 0$ almost everywhere if and only if $f \geq 0$ almost everywhere. The bipositive mappings between the spaces of multipliers are defined analogously.

Gaudry [3] proved that if there is a bipositive or isometric isomorphism between the multiplier spaces of $L_{p}\left(G_{1}\right)$ and $L_{p}\left(G_{2}\right)$, $l \leq p<\infty$ and $p \neq 2$, then $G_{1}$ and $G_{2}$ are topologically isomorphic. There has been considerable interest in results where the existence of some kind of isomorphism between spaces of functions on $G_{1}$ and $G_{2}$ or between their multiplier spaces implies that $G_{1}$ and $G_{2}$ are topologically isomorphic. See, Edwards [2], Johnson [6], Nagrajan [9], Parrot [10], Rigelhof [14], Strichartz [15], [16] and Tewari [17]. In this paper we consider isomorphisms between Segal algebras $S_{1}, S_{2}$ on $G_{1}, G_{2}$ or 
between their multiplier algebras and under some conditions on $S_{1}, S_{2}, G_{1}$, $G_{2}$ we prove that $G_{1}$ and $G_{2}$ are topologically isomorphic. Tewari [17] and Nagrajan [9] have proved special cases of our results. We also note that our proofs are simpler than those of Tewari and Nagrajan. Following are the main results of this paper.

THEOREM 1. Let $G_{1}, G_{2}$ be locally compact groups and let $S_{1}, S_{2}$ be Segal algebras on $G_{1}, G_{2}$ respectively. Suppose that any one of the following holds:

(i) $G_{1}$ and $G_{2}$ are abelian;

(ii) $G_{1}$ and $G_{2}$ are compact and $S_{1}$ and $S_{2}$ are symmetric;

(iii) $S_{1}$ and $S_{2}$ are pseudosymmetric.

If there is a bipositive isomorphism $\Psi$ of $M\left(S_{1}\right)$ onto $M\left(S_{2}\right)$ then $G_{1}$ and $G_{2}$ are topologically isomorphic.

THEOREM 2. Let $G_{1}, G_{2}$ be locally compact groups and let $S_{1}, S_{2}$ be Segal algebras on $G_{1}, G_{2}$ respectively. Suppose that the following conditions hold:

(i) either $G_{1}, G_{2}$ are abelian or $G_{1}, G_{2}$ are compact and $S_{1}, S_{2}$ are symmetric;

(ii) the isometric multipliers of $S_{1}$ and $S_{2}$ are just unimoduzar multiples of left translation operators.

If there is an isometric isomorphism $\Psi$ of $M\left(S_{1}\right\}$ onto $M\left(S_{2}\right)$ then $G_{1}$ and $G_{2}$ are topologically isomorphic.

\section{Bipositive isomorphisms}

In this section our main aim is to give a proof of Theorem 1 . We begin by proving a result about positive maltipliers of a Segal algebra.

LEMMA 1. Let $T$ be a positive multiplier of a Segal algebra $S$ on a locally compact group $G$. Suppose that any one of the following 
conditions holds:

(i) $G$ is abelian;

(ii) $G$ is compact and $S$ is symmetric;

(iii) $S$ is pseudosymmetric.

Then there exists a positive measure $\mu \in M(G)$ such that $T f=\mu$ *f for every $f \in S$.

Proof. Each of the conditions (i), (ii) and (iii) implies that there exists a two-sided approximate identity $\left\{f_{\alpha}\right\}$ of $S$ such that $f_{\alpha} \in S$, $f_{\alpha} \geq 0$ and $\left\|f_{\alpha}\right\|_{1}=1 ;$ see Theorems 33.12 and 28.53 of [5] and Proposition 1 on page 24 of [13]. Fixing $g \in S$ with $g \geq 0$ and $\|g\|_{1}=1$, we get

$$
\begin{aligned}
\left\|T f_{\alpha}\right\|_{1}=\left\|T f_{\alpha} * g\right\|_{1} \leq\left\|T f_{\alpha} * g\right\|_{S} & =\left\|T\left(f_{\alpha} * g\right)\right\|_{S} \\
& \leq\|T\|\left\|f_{\alpha} * g\right\|_{S} \\
& \leq\|T\| \|_{S} .
\end{aligned}
$$

The first equality occurs because $T f_{\alpha}$ and $g$ are non-negative and the last inequality follows because $\left\|f_{\alpha}\right\|_{1}=1 .\left\{T f_{\alpha}\right\}$ is now a bounded net in $M(G)$. Hence there is a subnet $\left\{T f_{\beta}\right\}$ and a $\mu \in M(G)$ such that $T f_{\beta}$ converges to $\mu$ in the weak star topology of $M(G)$. Since each $T f_{B}$ is non-negative, $\mu$ is a positive measure. We shall show that $T f=\mu * f$ for every $f \in S$.

Since $T f_{B}$ converges to $\mu$ in the weak star topology of $M(G)$, $T f_{B} * h(x)$ converges to $\mu * h(x)$ for every $h \in C_{0}(G)$ and $x \in G$. In particular, $T f_{\beta} * f * h(x)$ converges to $\mu * f * h(x)$ for every $f \in S$, $h \in C_{0}(G)$ and $x \in G$.

On the other hand, for any $f \in S, f_{\beta} * f$ converges to $f$ in $S$. Therefore $T\left(f_{\beta} * f\right)=T f_{\beta} * f$ converges in $S$, and hence in $L_{1}$, to $T f$. In particular, $T f_{\beta} * f$ converges to $T f$ in the weak star topology of $U(G)$ and hence $T f_{\beta} * f * h(x)$ converges to $T f * h(x)$ for every 
$h \in C_{0}(G)$ and $x \in G$. Thus $T f * h=\mu * f * h$ for every $f \in S$ and $h \in C_{0}(G)$. This implies that $T f=\mu * f$ for every $f \in S$ and the proof of the lemma is complete.

Proof of Theorem 1. Let $\Psi$ be a bipositive isomorphism of $M\left(S_{1}\right)$ onto $M\left(S_{2}\right)$ and let $\mu$ be a positive measure in $M\left(G_{1}\right)$. It follows that $\Psi_{\mu}$ is a positive multiplier of $S_{2}$ and, by Lemma 1 , we conclude that $\Psi \mu$ is a positive measure in $M\left(G_{2}\right)$. Conversely, every positive measure in $M\left(G_{2}\right)$ is the image under $\Psi$ of a positive multiplier of $S_{1}$, that is, of a positive measure in $M\left(G_{1}\right)$.

Thus $\Psi$ maps the positive cone of $M\left(G_{1}\right)$ onto that of $M\left(G_{2}\right)$. But any measure is a linear combination of positive measures. Hence $\Psi$ restricted to $M\left(G_{1}\right)$ is a bipositive isomorphism of $M\left(G_{1}\right)$ onto $M\left(G_{2}\right)$. It follows from the $L_{1}$-case of Theorem 2 of Gaudry [3] that $G_{1}$ and $G_{2}$ are topologically isomorphic.

REMARKS. I. In the abelian case Theorem 1 was proved by Tewari for multipliers of $A_{p}$-algebras. Nagrajan [9] modified the arguments of Tewari to prove the abelian case of Theorem 1 . Our proof is simpler even in the general case.

2. It is obvious that our proof of Theorem $I$ is valid for all pairs of Segal algebras $S_{1}, S_{2}$ whose positive multipliers are given by positive measures. The conditions in Theorem 1 are used only to ensure this; see Lemma 1 .

3. The proof of Theorem 1, given here, has the further merit that it applies to more general situations than those considered in Theorem 1 . For example, it holds good for any space of functions on the group whose multiplier algebra contains the measure algebra and all of whose positive multipliers are given by positive bounded measures. This is the case, for instance, for the $L_{p}$-spaces on a large class of groups, which include all abelian groups and compact groups; see Brainerd and Edwards [1]. However, it does not hold universally; see [3]. For those groups, for which it is 
true, our arguments will yield a simpler proof of Theorem 2 of [3] for $p>1$.

Rigelhof [14] proved a result about bipositive homomorphisms of measure algebras which contains Gaudry's $L_{1}$-case of Theorem 2 [3] as a special case. If, in the proof of Theorem 1, we use Rigelhof's result, we get the following more general version of Theorem 1. In view of Remark 3 above, it follows that a more general version of Gaudry's Theorem 2 [3] is true for certain groups which include all abelian groups and compact groups.

THEOREM 1'. Let $G_{1}, G_{2}$ and $S_{1}, S_{2}$ be as in Theorem 1. Suppose that there is a bipositive homomorphism $\Psi$ of $M\left(S_{1}\right)$ onto $M\left(S_{2}\right)$ such that if $\Psi\left(\mu * M\left(G_{1}\right)\right)=0$ for some $\mu \in M\left(G_{1}\right)$ then $\Psi(\mu)=0$. Then there is an open continuous homomorphism a of $G_{1}$ onto $G_{2}$. If $\Psi$ is an isomorphism then so is $\alpha$.

COROLLARY 1. Let $G_{1}, G_{2}$ and $S_{1}, S_{2}$ be as in Theorem 1. If there is a bipositive isomorphism $\Psi$ of $S_{1}$ onto $S_{2}$, then $G_{1}$ and $G_{2}$ are topologically isomorphic.

Proof. The map $T \rightarrow \Psi T \Psi^{-1}$ is a bipositive isomorphism of $M\left(S_{1}\right)$ onto $M\left(S_{2}\right)$ and the result follows from Theorem 1 .

REMARK. Corollary 1 , in the case of group algebras, was proved by Kawada [7]. His result was, perhaps, the first of the isomorphism theorems of the sort discussed in this paper. When $G_{1}, G_{2}$ are compact, corollary 1 was proved for the algebras $L_{p}\left(G_{i}\right)$ and $C\left(G_{i}\right), i=1,2$, by Edwards [2].

\section{Isometric isomorphisms}

Proof of Theorem 2. In the proof to follow, the multiplier corresponding to a measure $\mu$ will be denoted by $T_{\mu}$ except when $\mu$ is a point mass at a point, in which case $\delta_{a}$ will denote both the point measure concentrated at $a$ as well as the corresponding multiplier. This 
is done in order to avoid the confusion which might arise by using the same symbol for the multiplier as well as the measure.

Condition ( $i i)$ in Theorem 2 implies that any isometric multiplier of $S_{1}$ or $S_{2}$ is of the form $\lambda \delta_{a}$ or $\lambda \delta_{b}$ where $|\lambda|=1$ and $a \in G_{1}$ and $b \in G_{2}$.

For any $a \in G_{1}, \Psi \delta_{a}$ is an isometric multiplier of $S_{2}$ and hence there exists an element $b=\phi(a)$ of $G_{2}$ and a complex number $\lambda(a)$ with $|\lambda(a)|=I$ such that $\Psi \delta_{a}=\lambda(a) \delta_{b}$. This follows from condition (ii) of the theorem. Thus we get a map $\phi: G_{1} \rightarrow G_{2}$ defined by $\phi(\alpha)=b$. It can be easily seen that $\phi$ is an isomorphism of $G_{1}$ onto $G_{2}$. If the continuity of $\phi$ is proved, then similar considerations using $\psi^{-1}$ would complete the proof.

So suppose that $\phi$ is not continuous. Then there is a net $\left\{a_{i}\right\}$ converging to the identity element $e_{1}$ of $G_{1}$ and a subnet $\left\{\phi\left(a_{j}\right)\right\}$ of $\left\{\phi\left(a_{i}\right)\right\}$ lying entirely outside some neighbourhood $W$ of the identity element $e_{2}$ of $G_{2}$.

Now $\left\{\Psi \delta_{a_{j}}\right\}$ is a bounded net in $M\left(G_{2}\right)$ and hence some subnet $\left\{\Psi \delta_{a_{k}}\right\}$ of $\left\{\Psi \delta_{a_{j}}\right\}$ converges in the weak star topology to a measure $\mu \in M\left(G_{2}\right)$. Let $A$ denote the set of all $h \in L_{1}\left(G_{2}\right)$ having compactly supported Fourier transform if $G_{2}$ is abelian and the set of all trigonometric polynomials if $G_{2}$ is compact. Then $\Psi T_{f}(h) \in A$ for every $h \in A$ and $f \in L_{1}\left(G_{1}\right)$. Hence, for any $h \in A$ and $f \in L_{1}\left(G_{1}\right)$,

$$
\Psi \delta_{a_{k}}\left(\Psi T_{f}(h)\right)(x) \rightarrow T_{\mu}\left(\Psi T_{f}(h)\right)(x) \text { for every } x \in G_{2} .
$$

But $a_{\hat{k}} \rightarrow e_{1}$, so that $\delta_{a_{k}} * f \rightarrow f$ in $L_{1}$ and hence $\delta_{a_{k} T_{f}} \rightarrow T_{f}$ in $M\left(S_{1}\right)$. This implies that $\Psi \delta_{a_{k}} \Psi T_{f} \rightarrow \Psi T_{f}$ in $M\left(S_{2}\right)$ and $\Psi \delta_{a_{k}} \Psi T_{f}(g) \rightarrow \Psi T_{f}(g)$ in $S_{2}$, and hence in the weak star topology of 
$M\left(G_{2}\right)$, for every $g \in S_{2}$. Therefore, we get

(2) $\Psi \delta_{a_{k}} \Psi T_{f}(g) * h(x) \rightarrow \Psi T_{f}(g) * h(x)$ for every $x \in G_{2}$ and $h \in A$.

(1) and (2) imply that

$$
T_{\mu} \Psi T_{\hat{J}}(g) * h=\Psi T_{f}(g) * h \text { for } g \in S_{2}
$$

and $h \in A$. Since $A$ is dense in $S_{2}$, (3) implies that

$$
T_{\mu} \Psi T_{f}=\Psi T_{f} \text {, for any } f \in L_{1}\left(G_{1}\right)
$$

so that $\Psi^{-1}{ } \mu^{T} f=T_{f}$, which in turn implies that $\Psi^{-1} T_{\mu}$ equals the identity on $L_{1} * S_{1}\left(=S_{1}\right)$. This gives $T_{\mu}=\delta_{e_{2}}$ and so $\mu=\delta_{e_{2}}$.

Let $V$ be a neighbourhood of $e_{2}$ in $G_{2}$ such that $V^{-}$is compact and $V^{-} \subset W$. Here $V^{-}$denotes the closure of $V$. Choose $f \in C_{0}\left(G_{2}\right)$ such that $f\left(e_{2}\right)=1$ and support of $f$ is contained in $W$. Since $\phi\left(a_{k}\right) \xi W$ for any $k$, we have

$$
f\left(\phi\left(a_{k}\right)\right)=0 \text {, for each } k \text {. }
$$

However $\Psi\left(\delta_{a_{k}}\right)=\lambda\left(a_{k}\right) \delta_{\phi\left(a_{k}\right)}$ and $\Psi \delta_{a_{k}} \rightarrow \delta_{e_{2}}$ in the weak star topology of $M\left(G_{2}\right)$ so that $\lambda\left(a_{k}\right) f\left(\phi\left(a_{k}\right)\right)+f\left(e_{2}\right)=1$. This contradicts (4). Therefore $\phi$ is continuous and the proof of the theorem is complete.

COROLLARY 2. Let $G_{1}, G_{2}$ and $S_{1}, S_{2}$ be as in Theorem 2. If there is an isometric isomorphism $\Psi$ of $S_{1}$ onto $S_{2}$, then $G_{1}$ and $G_{2}$ are topologically isomorphic.

Proof. The map $T \rightarrow \Psi T \Psi^{-1}$ is an isometric isomorphism of $M\left(S_{1}\right)$ onto $i\left(S_{2}\right)$ and the result follows from Theorem 2 .

REMARKS. I. For a large class of Segal algebras which satisfy conditions (i) and (ii) of the theorem, see [11]. Without some conditions of the sort given in Theorem 2, the results are no longer valid; see Gaudry [3]. If $G_{1}=\pi, G_{2}=\pi \times \pi, S_{i}=L_{2}\left(G_{i}\right), i=1,2$, neither 
the theorem nor the corollary holds.

2. The proof given here will hold good on arbitrary locally compact groups if one can show that there is. a subspace $A$ of $S \cap C_{0}$ such that $A$ is invariant under all multipliers of $S$ and $A$ is either dense in $S$ or in $C_{0}$.

3. If it is assumed only that $\Psi$ is norm decreasing, $\Psi \delta_{a}$ is still an isometry (it has a norm decreasing inverse) and the techniques in the proof of Theorem 2 yield a continuous isomorphism of $G_{1}$ into $G_{2}$. The difficulty now appears to be in showing that this isomorphism is surjective. Rigelhof [14] has proved that the existence of a norm decreasing isomorphism between $M\left(G_{1}\right)$ and $M\left(G_{2}\right)$ implies that $G_{1}$ and $G_{2}$ are topologically isomorphic.

\section{References}

[1] 8. Brainerd and R.E. Edwards, "Linear operators which commute with translations. Part I: Representation theorems", J. Austral. Math. Soc. 6 (1966), 289-327.

[2] R.E. Edwards, "Bipositive and isometric isomorphisms of some convolution algebras", Canad. J. Math. 17 (1965), 839-846.

[3] G.1. Gaudry, "Isomorphism of multiplier algebras", Canad. J. Math. 20 (1968), 1165-1172.

[4] Henry Helson, "Isomorphisms of abelian group algebras", Ark. Mat. 2 $(1954), 475-487$.

[5] Edwin Hewitt and Kenneth A. Ross, Abstract harmonic analysis, Volume II (Die Grundlehren der mathematischen Wissenschaften, 152. Springer-Verlag, Berlin, Heidelberg, New York, 1970).

[6] B.E. Johnson, "Isometric isomorphisms of measure algebras", Proc. Amer. Math. Soc. 15 (1964), 186-188.

[7] Yukiyoshi Kawada, "On the group ring of a topological group", Math. Japon. 1 (1948), 1-5. 
[8] Ronald Larsen, An introduction to the theory of multipliers (Die Grundlehren der mathematischen Wissenschaften, 175. SpringerVerlag, Berlin, Heidelberg, New York, 1971).

[9] Kasturi Nagrajan, "Multipliers of Segal algebras" (Doctoral thesis, Institute of Mathematical Sciences, Madras, 1976).

[10] S.K. Parrot, "Isometric multipliers", Pacific J. Math. 25 (1968), $159-166$.

[11] K. Parthasarathy and U.B. Tewari, "Isometric multipliers of Segal algebras", Bulz. Austral. Math. Soc. 20 (1979), 105-114.

[12] Hans Reiter, Classical harmonic analysis and locally compact groups (Clarendon Press, London, 1968).

[13] Hans Reiter, $L^{1}$-algebras and Segal algebras (Lecture Notes in Mathematics, 231. Springer-Verlag, Berlin, Heidelberg, New York, 1971).

[14] Roger Rigelhof, "Norm decreasing homomorphisms of measure algebras", Trans. Amer. Math. Soc. 136 (1969), 361-371.

[15] Robert S. Strichartz, "Isometric isomorphisms of measure algebras", Pacific J. Math. 15 (1965), 315-317.

[16] Robert S. Strichartz, "Isomorphisms of group algebras", Proc. Amer. Math. Soc. 17 (1966), 858-862.

[17] U.B. Tewari, "Isomorphisms of some convolution algebras and their multiplier algebrasi", Bulz. Austral. Math. Soc. 7 (1972), $321-335$.

[18] J.G. Wendel, "On isometric isomorphism of group algebras", Pacific J. Math. 1 (1951), 305-311.

[19] J.G. Wendel, "Left centralizers and isomorphisms of group algebras", Pacific J. Math. 2 (1952), 251-261.

Department of Mathematics,

Indian Institute of Technology,

Kanpur 208016 , U.P.,

India.
Department of Mathematics, University of Calicut, Cal icut 673635, India. 